\title{
Peripheral Blood Lymphocyte-to-Monocyte Ratio as a Useful Prognostic Factor in Newly Diagnosed Multiple Myeloma
}

\author{
Ying Tian $\mathbb{D}^{1},{ }^{1}$ Yue Zhang $\left(\mathbb{D},{ }^{1,2}\right.$ Wan-Qiu Zhu $(\mathbb{D}),{ }^{1}$ Xiao-Lei Chen, ${ }^{2}$ \\ He-Bing Zhou (iD), ${ }^{2}$ and Wen-Ming Chen (iD) ${ }^{1}$ \\ ${ }^{1}$ Department of Hematology, Beijing Chao-Yang Hospital, Capital Medical University, Beijing, China \\ ${ }^{2}$ Department of Hematology, Beijing Lu-He Hospital, Capital Medical University, Beijing, China \\ Correspondence should be addressed to He-Bing Zhou; zhbyffs@126.com and Wen-Ming Chen; xybxx@ccmu.edu.cn
}

Received 24 July 2018; Revised 7 November 2018; Accepted 12 November 2018; Published 26 November 2018

Academic Editor: Ruxana Sadikot

Copyright (c) 2018 Ying Tian et al. This is an open access article distributed under the Creative Commons Attribution License, which permits unrestricted use, distribution, and reproduction in any medium, provided the original work is properly cited.

\begin{abstract}
The survival of individuals with tumors may be predicted by the peripheral blood lymphocyte-to-monocyte ratio (LMR) upon diagnosis in recent studies. For patients with multiple myeloma (MM) in the era of novel agents, the prognostic significance of LMR remains unclear. In this study, the prognostic impact of LMR is evaluated by 285 patients with MM who are treated with proteasome inhibitor and/or immunomodulatory drug. LMR is a proven predictor of survival using the receiver operating characteristic curve, with 4.2 as the cutoff point. Patients with LMR $\leq 4.2$ at diagnosis had poorer overall survival (OS) and progression-free survival (PFS) than those with LMR > 4.2. In addition, multivariate analysis showed that LMR less than 4.2 is an independent predictor for the OS (hazard ratio [HR]: 1.703; 95\% confidence interval [CI]: $1.020-2.842 ; P=0.042$ ) and PFS (HR: $1.831 ; 95 \%$ CI: $1.098-3.053$; $P=0.021$ ). According to the test, the LMR at diagnosis, which functions as a simple index reflecting host systemic immunity, can predict clinical outcomes in patients with MM who are treated with new agents.
\end{abstract}

\section{Introduction}

Multiple myeloma (MM) is the second most common hematological malignancy, which accounts for nearly $10 \%$ of all hematological malignant disorders and $0.9 \%$ of all cancer deaths each year $[1,2]$. For newly diagnosed MM patients, the proteasome inhibitor and/or immunomodulatory drugbased chemotherapy is combined with autologous stem cell transplantation (ASCT) if eligible. Outcome of patients with MM was improved with these new therapies [3]. However, it is worth noting that MM remains incurable, and accurate and practical prognostic indicators for predicting survival will be used for MM patients.

At present, the International Staging System (ISS) [4] is the primary tool to predict the prognosis of MM patients. The Revised-International Staging System (R-ISS) was developed as a newly revised tool in which cytogenetics and lactate dehydrogenase (LDH) are used as the prognostic factors that are independent of the ISS staging system [5]. The R-ISS is proven effective in improving the stratification of patients into more homogeneous risk groups, and it has a better discriminative power than the ISS for MM patients who were treated with novel agents as the primary therapy [6].

The immune system is an important factor in tumor pathogenesis. Immune biomarkers which reflect the baseline host immune status, for example, lymphocyte-to-monocyte ratio (LMR) may be related to the poor prognosis of various tumors [7]. Shi has reported that LMR is associated with the survival of MM patients and is an independent prognostic factor for OS [8]. However, studies on LMR for newly diagnosed MM patients who were treated with novel agents are limited.

This study aimed to investigate the prognostic value of peripheral blood LMR, combined with iFISH in newly diagnosed MM patients.

\section{Materials and Methods}

2.1. Patients. 285 patients with newly diagnosed MM from the Beijing Chao-Yang Hospital, Capital Medical University, 
were studied from June 1, 2010 to December 31, 2016. All patients were diagnosed according to the IMWG diagnostic criteria 2014 [9]. The baseline data from the patients were collected: age at diagnosis; gender; complete blood count (CBC); serum albumin, LDH, creatinine, calcium, and $\beta 2$ microglobulin levels; and iFISH analysis including 1q21 gain, del $(17 \mathrm{p}), \mathrm{t}(4 ; 14), \mathrm{t}(11 ; 14)$, and $\mathrm{t}(14 ; 16)$. The LMR was calculated by peripheral blood white cell count.

The study was censored on December 31, 2017. The study was approved by the ethics committee of Beijing Chao-Yang Hospital.

2.2. Statistical Analysis. All patients were treated with at least one novel agent, followed by ASCT if eligible. During the study, the patients were evaluated for CBC, serum albumin, LDH, creatinine, calcium, and $\beta 2$-microglobulin, serum and urine $\mathrm{M}$ protein, bone marrow and multiparametric flow cytometry every 3 months. The overall survival (OS) is defined as the time from diagnosis to death from any cause. Progression-free survival (PFS) was calculated from the date of diagnosis to the date of disease progression, death from any cause, or the last contact, whichever occurred first. Kaplan-Meier analysis was conducted to estimate the survival of patients, and log-rank test was used for comparison. $P$ values $<0.05$ were considered statistically significant. The $\chi^{2}$ test (nonparametric analysis) was adopted to evaluate the differences between groups. In the multivariate analysis, a Cox proportional hazards model was used. Receiver operating characteristic curves (ROC) and area under the curve (AUC) were used to determine the best cutoff values for survival that is indicated by the LMR. A statistical analysis was performed using the IBM Statistical Package for the Social Sciences software (SPSS Inc, Chicago, IL, USA) or R 3.3.1 software (Institute for Statistics and Mathematics, Vienna, Austria; http://www.r-project.org/).

\section{Results}

3.1. Characteristics of the Patients. In this retrospective analysis 285 newly diagnosed MM patients, baseline clinical and laboratory characteristics are listed in Table 1 . The median follow-up duration was 48 (range: 2-84) months. The 3-year OS and PFS of the entire cohort were $69.8 \%$ and $50.0 \%$, respectively.

The median LMR at diagnosis was 6.18 (range: 0.60-118.00). Using the data from the entire cohort, we selected the cutoff points of the LMR for predicting the survival outcomes in the ROC curve analysis. The most discriminative cutoff value of LMR was 4.2, with an AUC value of 0.607 (95\% confidence interval [CI]: 0.529-0.685; Figure 1).

Table 1 shows the patients' characteristics according to the LMR at diagnosis. Patients with a LMR $\leq 4.2$ had elevated levels of serum $\mathrm{LDH}(P<0.001)$, creatinine $(P=0.006)$, calcium $(P=0.003)$, and $\beta 2$-microglobulin $(P=0.035)$, and they had a higher incidence of relapse $(P=0.001)$ than those with a LMR $>4.2$. The results also showed that patients with LMR $>4.2$ had a higher incidence of $t(11 ; 14)$ than those with LMR $\leq 4.2(24.8 \%$ vs. $13.6 \%, P=0.018)$.

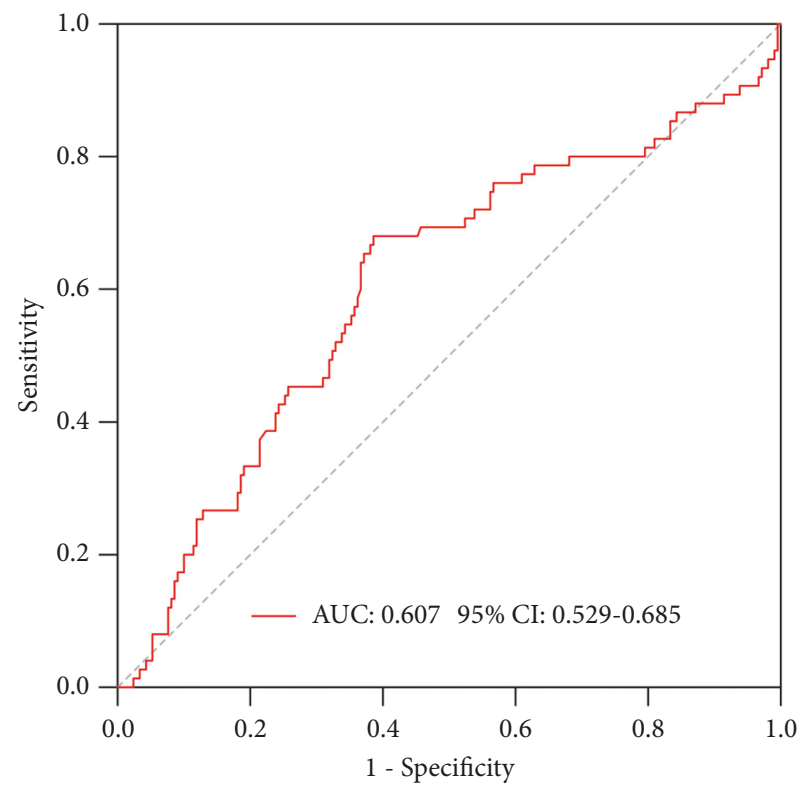

FIgURE 1: ROC and AUC for the LMR at diagnosis for MM patients. Abbreviations: AUC, area under the curve; LMR, lymphocyte-tomonocyte ratio; MM, multiple myeloma; ROC, receiver operating characteristic.

3.2. Prognostic Impact of the Lymphocyte/Monocyte Ratio at Diagnosis. The OS and PFS of patients with LMR $\leq 4.2$ were significantly lower than those of patients with LMR $>4.2$ at diagnosis (3-year OS: $64.2 \%$ vs. 77.3\%, $P=0.001$; 3-year PFS: $37.9 \%$ vs. $68.1 \%, P<0.001$; Figure 2 ).

Tables 2 and 3 showed the results of the univariate and multivariate analysis of the factors influencing the OS and PFS, respectively. The multivariate analysis revealed that LMR $\leq 4.2$ was an independent prognostic factor for predicting OS (HR: 1.703; 95\% CI: 1.020-2.842; $P=0.042$; Table 2) and PFS (HR: 1.831; 95\% CI: 1.098-3.053; $P=0.021$; Table 3). Moreover, serum $\beta 2$-microglobulin $\geq 5.5 \mathrm{mmol} / \mathrm{L}$ was also an independent prognostic factor for OS (HR: 1.810; 95\% CI: 1.049-3.125; $P=0.033$; Table 2 ) and PFS (HR: 1.758 ; 95\% CI: 1.014-3.047; $P=0.044$; Table 3).

3.3. Prognostic Impact of the Lymphocyte/Monocyte Ratio on Different ISS and R-ISS. Further analysis showed that LMR $\leq 4.2$ had a negative prognostic impact on both PFS and OS in ISS stages II and III, while it could not in ISS stage I (see Figure 3). Moreover, LMR $\leq 4.2$ had a negative prognostic impact on both PFS and OS in R-ISS stage II, while it could not in R-ISS stages I and III (see Figure 4).

\section{Discussion}

As a marker of host antitumor immunity, absolute lymphocyte count (ALC) has been widely studied in hematologic and solid malignancies [10]. In MM, ALC recovery after ASCT has significant prognostic value $[11,12]$. Moreover, another study has shown that ALC at diagnosis was associated with survival in patients with newly diagnosed MM [13]. 
TABLE 1: Characteristics at baseline of 285 newly diagnosed MM patients.

\begin{tabular}{|c|c|c|c|c|}
\hline Characteristic & All cases $(n=285)$ & $\mathrm{LMR} \leqslant 4.2(\mathrm{n}=132)$ & LMR $>4.2(\mathrm{n}=153)$ & $P$ \\
\hline Age at diagnosis, years & & & & 0.478 \\
\hline$<65$ & 197 & 94 & 103 & \\
\hline$\geqslant 65$ & 88 & 38 & 50 & \\
\hline Gender & & & & 0.200 \\
\hline Male & 159 & 79 & 80 & \\
\hline Female & 126 & 53 & 73 & \\
\hline Hemoglobin & & & & 0.878 \\
\hline$\geqslant 100 \mathrm{~g} / \mathrm{L}$ & 126 & 59 & 67 & \\
\hline$<100 \mathrm{~g} / \mathrm{L}$ & 159 & 73 & 86 & \\
\hline Serum albumin & & & & 0.073 \\
\hline$\geqslant 35 \mathrm{~g} / \mathrm{L}$ & 101 & 54 & 47 & \\
\hline$<35 \mathrm{~g} / \mathrm{L}$ & 184 & 78 & 106 & \\
\hline Serum LDH & & & & 0.001 \\
\hline$<250 \mathrm{U} / \mathrm{L}$ & 236 & 96 & 140 & \\
\hline$\geqslant 250 \mathrm{U} / \mathrm{L}$ & 49 & 36 & 13 & \\
\hline Serum creatinine & & & & 0.006 \\
\hline$<177 \mathrm{umol} / \mathrm{L}$ & 235 & 100 & 135 & \\
\hline$\geqslant 177 \mathrm{umol} / \mathrm{L}$ & 50 & 32 & 18 & \\
\hline Serum calcium & & & & 0.003 \\
\hline$<2.75 \mathrm{mmol} / \mathrm{L}$ & 266 & 117 & 149 & \\
\hline$\geqslant 2.75 \mathrm{~mol} / \mathrm{L}$ & 19 & 15 & 4 & \\
\hline Serum $\beta 2$-microglobulin & & & & 0.035 \\
\hline$<5.5 \mathrm{mg} / \mathrm{L}$ & 172 & 71 & 101 & \\
\hline$\geq 5.5 \mathrm{mg} / \mathrm{L}$ & 113 & 61 & 52 & \\
\hline 1q21 gain & & & & 0.851 \\
\hline Negative & 155 & 71 & 84 & \\
\hline Positive & 130 & 61 & 69 & \\
\hline $\operatorname{del}(17 p)$ & & & & 0.790 \\
\hline Negative & 252 & 116 & 136 & \\
\hline Positive & 33 & 16 & 17 & \\
\hline $\mathrm{t}(4 ; 14)$ & & & & 0.080 \\
\hline Negative & 246 & 119 & 127 & \\
\hline Positive & 39 & 13 & 26 & \\
\hline $\mathrm{t}(11 ; 14)$ & & & & 0.018 \\
\hline Negative & 229 & 114 & 115 & \\
\hline Positive & 56 & 18 & 38 & \\
\hline $\mathrm{t}(14 ; 16)$ & & & & 0.953 \\
\hline Negative & 274 & 127 & 147 & \\
\hline Positive & 11 & 5 & 6 & \\
\hline Relapse & & & & 0.001 \\
\hline No & 176 & 70 & 106 & \\
\hline Yes & 83 & 51 & 32 & \\
\hline \multicolumn{5}{|l|}{ ASCT } \\
\hline No & 196 & 92 & 104 & 0.798 \\
\hline Yes & 89 & 40 & 49 & \\
\hline
\end{tabular}

Abbreviations: ASCT, autologous stem cell transplantation; LDH, lactate dehydrogenase; LMR, lymphocyte-to-monocyte ratio; MM, multiple myeloma. 
TABLE 2: Univariate and multivariate analyses for OS.

\begin{tabular}{|c|c|c|c|c|}
\hline \multirow{2}{*}{ Prognostic factors } & \multicolumn{2}{|c|}{ Univariate analysis } & \multicolumn{2}{|c|}{ Multivariate analysis } \\
\hline & $\operatorname{HR}(95 \% \mathrm{CI})$ & $P$ & $\operatorname{HR}(95 \% \mathrm{CI})$ & $P$ \\
\hline Age $\geq 65$ years & $1.045(0.630-1.733)$ & 0.866 & & \\
\hline Hemoglobin $<100 \mathrm{~g} / \mathrm{L}$ & $1.305(0.820-2.078)$ & 0.262 & & \\
\hline Serum albumin $<35 \mathrm{~g} / \mathrm{L}$ & $1.381(0.840-2.272)$ & 0.203 & & \\
\hline Serum $\mathrm{LDH} \geq 250 \mathrm{U} / \mathrm{L}$ & $2.748(1.661-4.548)$ & $<0.001$ & $1.775(0.984-3.199)$ & 0.056 \\
\hline Serum creatinine $\geq 177 \mathrm{umol} / \mathrm{L}$ & $1.953(1.178-3.238)$ & 0.009 & $1.138(0.591-2.192)$ & 0.699 \\
\hline Serum calcium $\geq 2.75 \mathrm{~mol} / \mathrm{L}$ & $2.761(1.371-5.559)$ & 0.004 & $1.427(0.657-3.102)$ & 0.369 \\
\hline Serum $\beta 2$-microglobulin $\geq 5.5 \mathrm{mmol} / \mathrm{L}$ & $2.410(1.523-3.815)$ & $<0.001$ & $1.810(1.049-3.125)$ & 0.033 \\
\hline 1q21 gain & $1.714(1.086-2.704)$ & 0.021 & $1.527(0.957-2.436)$ & 0.076 \\
\hline $\operatorname{del}(17 p)$ & $1.526(0.803-2.902)$ & 0.197 & & \\
\hline $\mathrm{t}(4 ; 14)$ & $1.009(0.501-2.033)$ & 0.980 & & \\
\hline $\mathrm{t}(11 ; 14)$ & $1.431(0.830-2.465)$ & 0.197 & & \\
\hline $\mathrm{t}(14 ; 16)$ & $1.910(0.695-5.246)$ & 0.210 & & \\
\hline $\mathrm{LMR} \leq 4.2$ & $2.207(1.356-3.591)$ & 0.001 & $1.703(1.020-2.842)$ & 0.042 \\
\hline
\end{tabular}

Abbreviations: CI, confidence interval; HR, hazard ratio; LDH, lactate dehydrogenase; LMR, lymphocyte-to-monocyte ratio; OS, overall survival

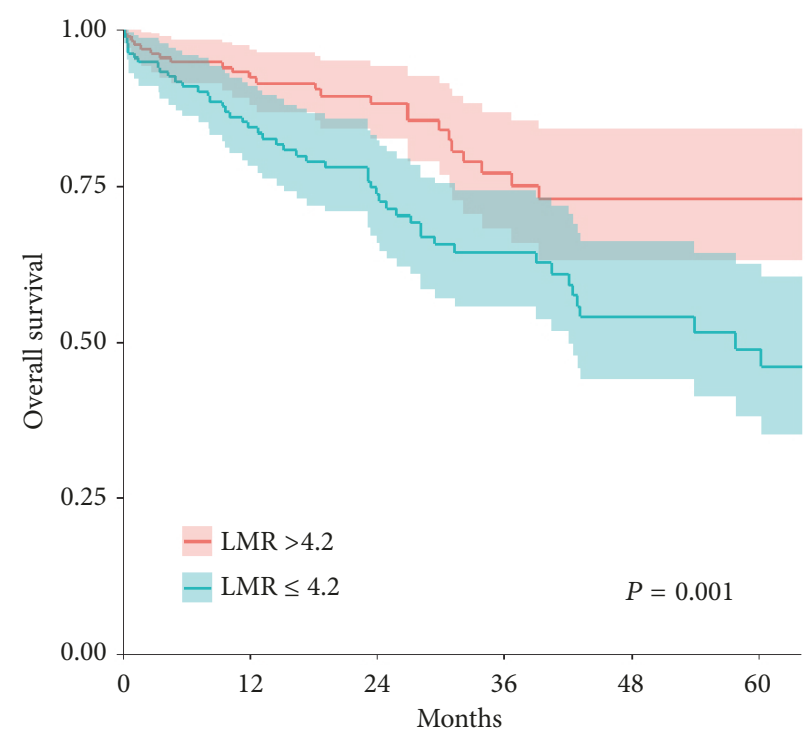

(a)

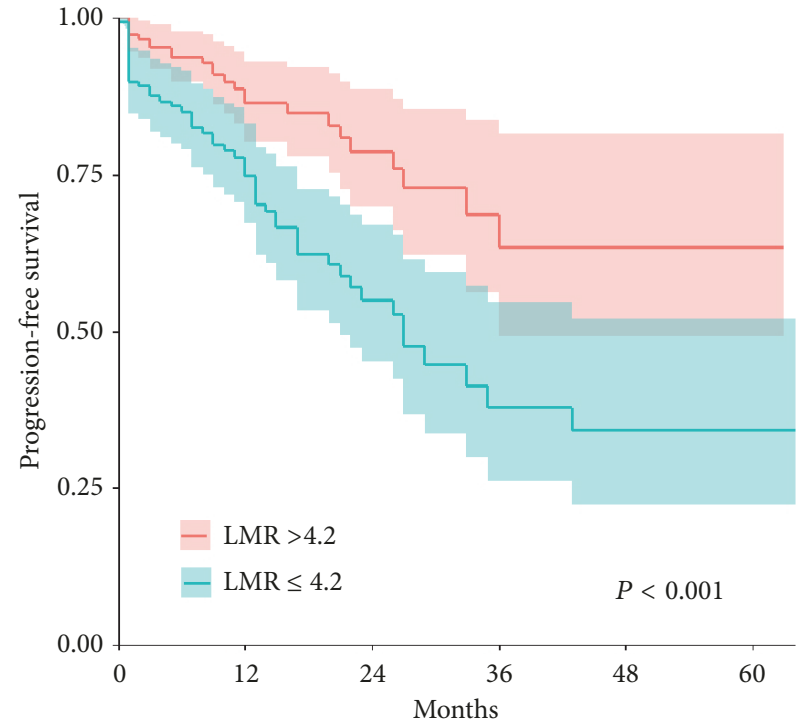

(b)

FIGURE 2: (a) Overall survival for the LMR at diagnosis for MM patients. (b) Progression-free survival for LMR at diagnosis for MM patients. Abbreviations: LMR, lymphocyte-to-monocyte ratio; MM, multiple myeloma.

The BM micro-environment plays a critical role in the development of MM from its precursor condition, monoclonal gammopathy of undetermined significance (MGUS), in part by allowing immune tumor evasion [14]. In addition, it can support the growth and survival of myeloma cells and influence their migration and drug resistance $[15,16]$. Inflammatory cells are essential in tumor progression $[17,18]$. Tumor-associated macrophages (TAMs), which constitute a significant proportion of tumor-related inflammatory cells, contribute to the growth, angiogenesis, and metastasis of a variety of tumors [19].

TAMs play an important prognostic role in patients with classical Hodgkin lymphoma (HL) [20-22], follicular lymphoma (FL) [23], and MM [24-26]. Derived from the circulating monocytes, TAMs are recruited to the tumor site by tumor-derived chemotactic factors [27]. Tumor-derived chemotactic factors can influence the number of circulating monocytes and the TAMs, the level of TAM recruitment can be reflected by the peripheral blood absolute monocyte count (AMC), and it may be considered as an important surrogate marker for TAMs. AMC is a poor prognostic factor in diffusing large B-cell lymphoma (DLBCL) [28], FL [29], extranodal natural killer/T-cell lymphoma (ENKL) [30], HL [31], and MM [24].

Based on these results, the ALC-to-AMC ratio may be considered as a symbol of the relative strength of the host 
TABLE 3: Univariate and multivariate analyses for PFS.

\begin{tabular}{|c|c|c|c|c|}
\hline \multirow{2}{*}{ Prognostic factors } & \multicolumn{2}{|c|}{ Univariate analysis } & \multicolumn{2}{|c|}{ Multivariate analysis } \\
\hline & $\operatorname{HR}(95 \% \mathrm{CI})$ & $P$ & $\operatorname{HR}(95 \% \mathrm{CI})$ & $P$ \\
\hline Age $\geq 65$ years & $1.113(0.671-1.844)$ & 0.679 & & \\
\hline Hemoglobin < 100g/L & $1.431(0.900-2.274)$ & 0.130 & & \\
\hline Serum albumin $<35 \mathrm{~g} / \mathrm{L}$ & $1.437(0.874-2.364)$ & 0.153 & & \\
\hline Serum $\mathrm{LDH} \geq 250 \mathrm{U} / \mathrm{L}$ & $2.820(1.696-4.687)$ & $<0.001$ & $1.673(0.941-2.973)$ & 0.080 \\
\hline Serum creatinine $\geq 177 \mathrm{umol} / \mathrm{L}$ & $2.113(1.275-3.503)$ & 0.004 & $1.005(0.530-1.904)$ & 0.989 \\
\hline Serum calcium $\geq 2.75 \mathrm{~mol} / \mathrm{L}$ & $2.996(1.489-6.032)$ & 0.002 & $1.499(0.685-3.278)$ & 0.311 \\
\hline Serum $\beta 2$-microglobulin $\geq 5.5 \mathrm{mmol} / \mathrm{L}$ & $2.541(1.604-4.024)$ & $<0.001$ & $1.758(1.014-3.047)$ & 0.044 \\
\hline 1q21 gain & $1.772(1.121-2.800)$ & 0.014 & $1.586(0.994-2.529)$ & 0.053 \\
\hline $\operatorname{del}(17 p)$ & $1.439(0.758-2.731)$ & 0.266 & & \\
\hline $\mathrm{t}(4 ; 14)$ & $1.079(0.535-2.176)$ & 0.831 & & \\
\hline $\mathrm{t}(11 ; 14)$ & $1.550(0.901-2.668)$ & 0.114 & & \\
\hline $\mathrm{t}(14 ; 16)$ & $1.638(0.579-4.496)$ & 0.338 & & \\
\hline $\mathrm{LMR} \leq 4.2$ & $2.387(1.469-3.880)$ & $<0.001$ & $1.831(1.098-3.053)$ & 0.021 \\
\hline
\end{tabular}

Abbreviations: CI, confidence interval; HR, hazard ratio; LDH, lactate dehydrogenase; LMR, lymphocyte-to-monocyte ratio; PFS, progression-free survival

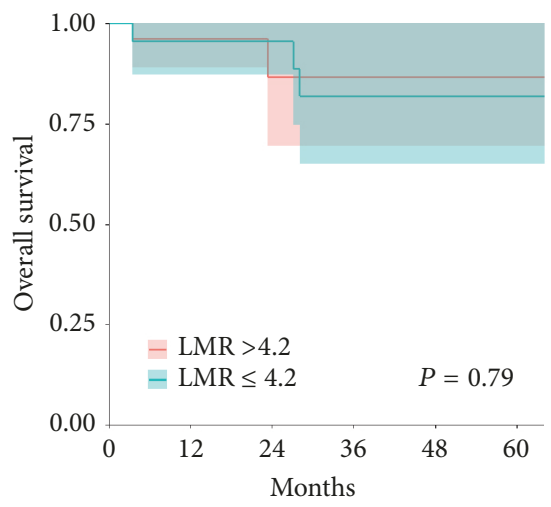

(a)

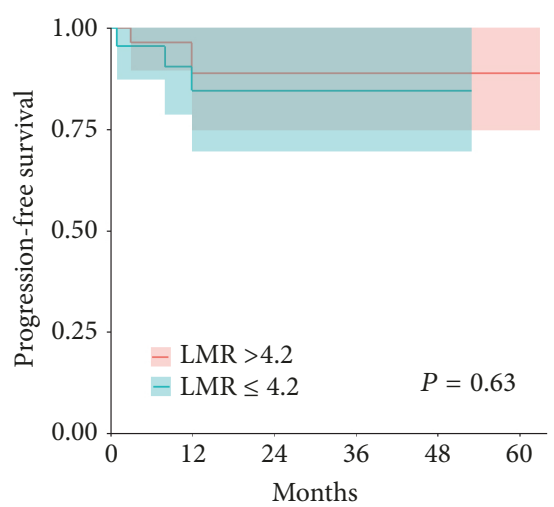

(d)

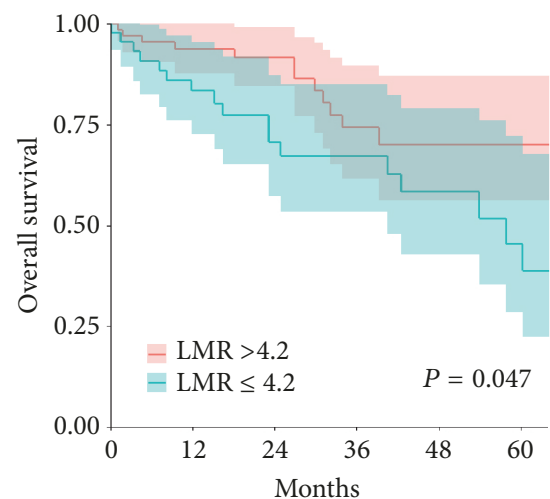

(b)

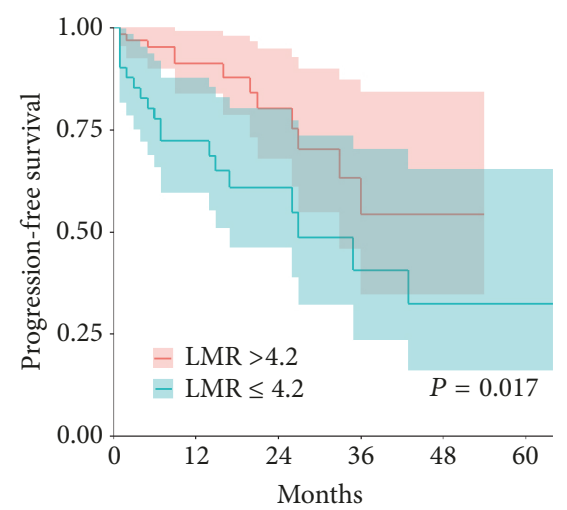

(e)

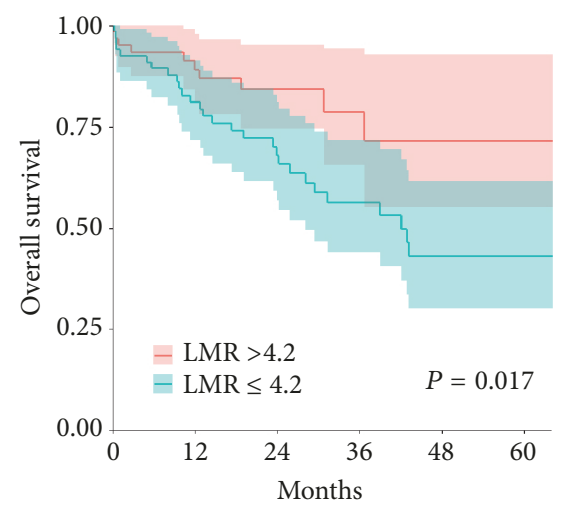

(c)

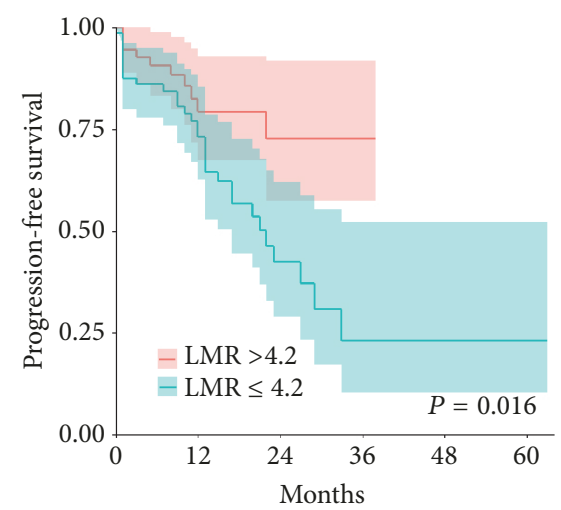

(f)

FIGURE 3: (a) Overall survival in ISS stage I stratified by the LMR. (b) Overall survival in ISS stage II stratified by the LMR. (c) Overall survival in ISS stage III stratified by the LMR. (d) Progression-free survival in ISS stage I stratified by the LMR. (e) Progression-free survival in ISS stage II stratified by the LMR. (f) Progression-free survival in ISS stage III stratified by the LMR.

immune system to tumor-induced immune dysfunction. A lower LMR has a negative prognostic impact on several solid tumors and hematologic malignancies [7, 32, 33]. A recent study has reported that LMR $<3.6$ can be considered a bad prognostic factor for PFS in patients with MM [34].
Therefore, LMR has been associated with the prognosis of patients with several tumors.

In this paper, the prognostic impact of the LMR in newly diagnosed MM patients was treated by proteasome inhibitor and/or immunomodulatory drug-based chemotherapy was 


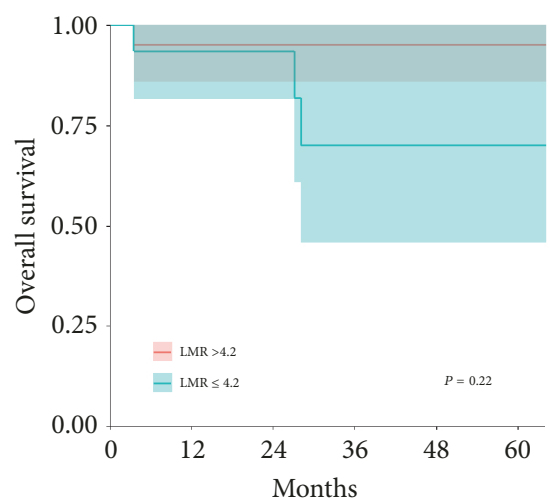

(a)

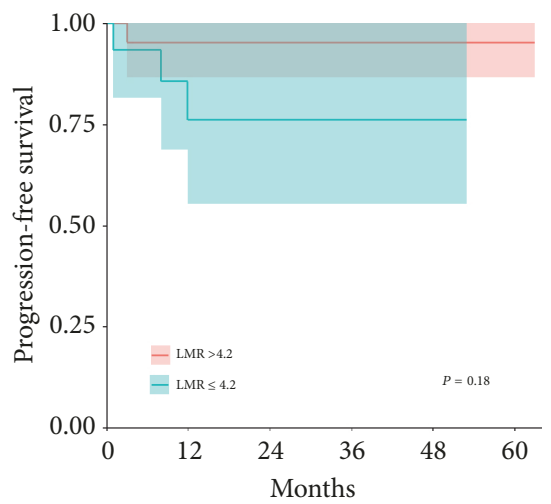

(d)

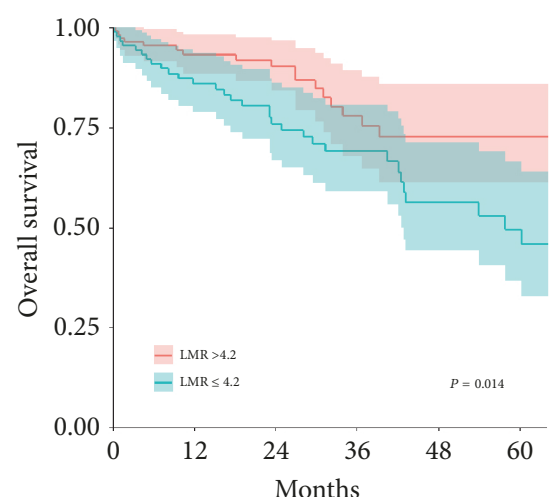

(b)

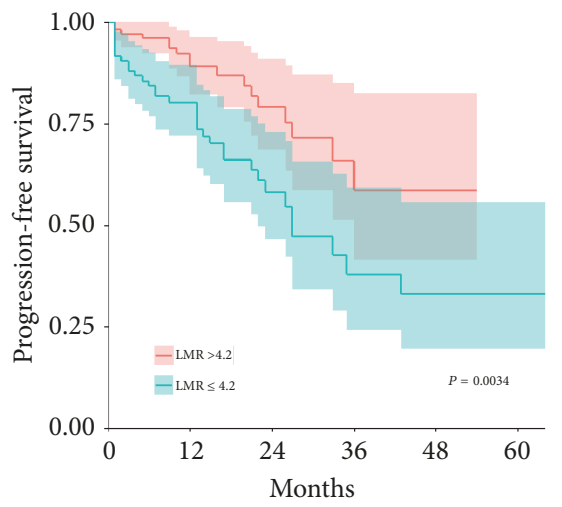

(e)

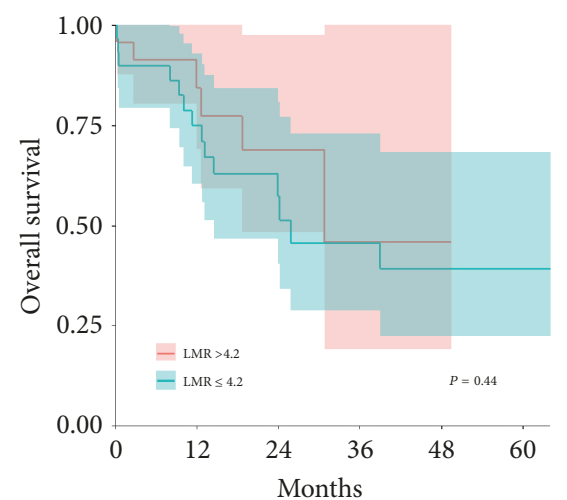

(c)

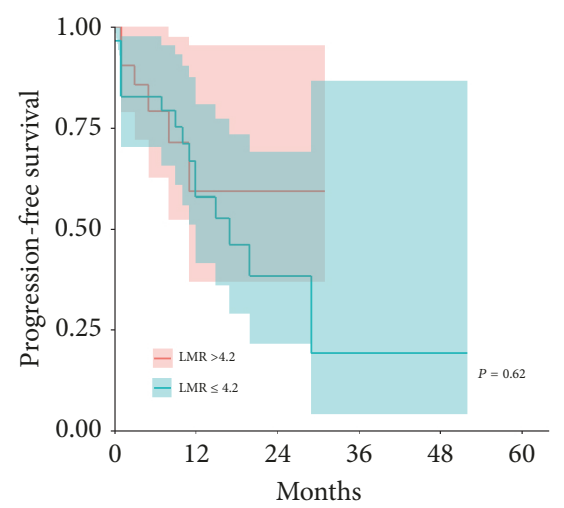

(f)

FIgure 4: (a) Overall survival in R-ISS stage I stratified by the LMR. (b) Overall survival in R-ISS stage II stratified by the LMR. (c) Overall survival in R-ISS stage III stratified by the LMR. (d) Progression-free survival in R-ISS stage I stratified by the LMR. (e) Progression-free survival in R-ISS stage II stratified by the LMR. (f) Progression-free survival in R-ISS stage III stratified by the LMR.

retrospectively evaluated. The result showed that LMR was an independent predictor for OS and PFS, and patients with LMR $\leq 4.2$ had significantly elevated serum LDH, creatinine, calcium, and $\beta 2$-microglobulin levels and a higher incidence of relapse. In addition, patients with LMR $>4.2$ had a higher incidence of $\mathrm{t}(11 ; 14)$. However, Dosani et al. have found that LMR independently predicted del $(17 \mathrm{p})$ and $t(4 ; 14)$, rather than $t(11 ; 14)$ [35]. This may be due to differences in the study population and may also be related to differences in the selected cutoff values. Thus, prospective studies with a larger sample size must be conducted to validate the results.

Considering the significance of LMR in clinical outcomes, certain tests have also been conducted to validate whether LMR could improve the prognostic impact of the ISS and R-ISS. Interestingly, based on the test results, the addition of LMR to the ISS further defined prognosis, particularly in stages II and III, whereas those to the R-ISS further defined prognosis in stage II. The combination of ISS or R-ISS and LMR improved the predictive value in patients treated with novel agents beforehand.

\section{Conclusions}

The LMR at diagnosis, which is a simple index reflecting host systemic immunity, can predict clinical outcomes in patients with MM who were treated with novel agents. In addition, LMR is an objective and cost-effective test result, and it can be easily obtained from CBC results. Owing to its significant value in clinical treatment, further studies on LMR must be conducted to better understand the roles of peripheral lymphocytes and monocytes in individuals with MM.

\section{Data Availability}

The data used to support the findings of this study are available from the corresponding author upon request.

\section{Conflicts of Interest}

The authors declare that they have no conflicts of interest.

\section{Authors' Contributions}

Ying Tian and Yue Zhang contributed equally to this work.

\section{Acknowledgments}

This study was supported by Beijing Municipal Natural Science Foundation (No. 7162068). 


\section{References}

[1] N. Becker, "Epidemiology of multiple myeloma," Recent results in cancer research Fortschritte der Krebsforschung Progres dans les recherches sur le cancer, vol. 183, pp. 25-35, 2011.

[2] S. Vincent Rajkumar, "Multiple myeloma: 2014 Update on diagnosis, risk-stratification, and management," American Journal of Hematology, vol. 89, no. 10, pp. 999-1009, 2014.

[3] A. Kumar, I. Hozo, K. Wheatley, and B. Djulbegovic, "Thalidomide versus bortezomib based regimens as first-line therapy for patients with multiple myeloma: A systematic review," American Journal of Hematology, vol. 86, no. 1, pp. 18-24, 2011.

[4] P. R. Greipp, J. S. Miguel, B. G. M. Dune et al., "International staging system for multiple myeloma," Journal of Clinical Oncology, vol. 23, no. 15, pp. 3412-3420, 2005.

[5] A. Palumbo, H. Avet-Loiseau, and S. Oliva, "Revised international staging system for multiple myeloma: a report from International Myeloma Working Group," Journal of Clinical Oncology, 2015.

[6] H. Cho, D. H. Yoon, J. B. Lee et al., "Comprehensive evaluation of the revised international staging system in multiple myeloma patients treated with novel agents as a primary therapy," American Journal of Hematology, vol. 92, no. 12, pp. 1280-1286, 2017.

[7] T. F. Nishijima, H. B. Muss, S. S. Shachar, K. Tamura, and Y. Takamatsu, "Prognostic value of lymphocyte-to-monocyte ratio in patients with solid tumors: a systematic review and metaanalysis," Cancer Treatment Reviews, vol. 41, no. 10, pp. 971-978, 2015.

[8] S.-J. Shin, J. Roh, M. Kim et al., "Prognostic significance of absolute lymphocyte count/absolute monocyte count ratio at diagnosis in patients with multiple myeloma," The Korean Journal of Pathology, vol. 47, no. 6, pp. 526-533, 2013.

[9] S. V. Rajkumar, M. A. Dimopoulos, and A. Palumbo, "International Myeloma Working Group updated criteria for the diagnosis of multiple myeloma," The Lancet Oncology, vol. 15, no. 12, pp. e538-e548, 2014.

[10] L. F. Porrata, K. Ristow, T. M. Habermann, T. E. Witzig, D. J. Inwards, and S. N. Markovic, "Absolute lymphocyte count at the time of first relapse predicts survival in patients with diffuse large B-cell lymphoma," American Journal of Hematology, vol. 84, no. 2, pp. 93-97, 2009.

[11] H. Kim, H.-J. Sohn, S. Kim, J.-S. Lee, W.-K. Kim, and C. Suh, "Early lymphocyte recovery predicts longer survival after autologous peripheral blood stem cell transplantation in multiple myeloma," Bone Marrow Transplantation, vol. 37, no. 11, pp. 1037-1042, 2006.

[12] L. F. Porrata, M. A. Gertz, D. J. Inwards et al., "Early lymphocyte recovery predicts superior survival after autologous hematopoietic stem cell transplantation in multiple myeloma or non-Hodgkin lymphoma," Blood, vol. 98, no. 3, pp. 579-585, 2001.

[13] H. Ege, M. A. Gertz, S. N. Markovic et al., "Prediction of survival using absolute lymphocyte count for newly diagnosed patients with multiple myeloma: A retrospective study," British Journal of Haematology, vol. 141, no. 6, pp. 792-798, 2008.

[14] P. Rodríguez-Otero, B. Paiva, M. Engelhardt, F. Prósper, and J. F. San Miguel, "Is immunotherapy here to stay in multiple myeloma?" Haematologica, vol. 102, no. 3, pp. 423-432, 2017.

[15] K. Podar, D. Chauhan, and K. C. Anderson, "Bone marrow microenvironment and the identification of new targets for myeloma therapy," Leukemia, vol. 23, no. 1, pp. 10-24, 2009.
[16] C. S. Mitsiades, N. S. Mitsiades, P. G. Richardson, N. C. Munshi, and K. C. Anderson, "Multiple myeloma: a prototypic disease model for the characterization and therapeutic targeting of interactions between tumor cells and their local microenvironment," Journal of Cellular Biochemistry, vol. 101, no. 4, pp. 950968, 2007.

[17] L. M. Coussens and Z. Werb, "Inflammation and cancer," Nature, vol. 420, no. 6917, pp. 860-867, 2002.

[18] F. Balkwill and A. Mantovani, "Inflammation and cancer: back to Virchow?" The Lancet, vol. 357, no. 9255, pp. 539-545, 2001.

[19] J. W. Pollard, "Tumour-educated macrophages promote tumour progression and metastasis," Nature Reviews Cancer, vol. 4, no. 1, pp. 71-78, 2004.

[20] T. Álvaro-Naranjo, M. Lejeune, M. T. Salvadó-Usach et al., “Tumor-infiltrating cells as a prognostic factor in Hodgkin's lymphoma: A quantitative tissue microarray study in a large retrospective cohort of 267 patients," Leukemia \& Lymphoma, vol. 46, no. 11, pp. 1581-1591, 2005.

[21] C. Steidl, T. Lee, and S. P. Shah, "Tumor-associated macrophages and survival in classic Hodgkin's lymphoma," The New England Journal of Medicine, vol. 362, no. 10, pp. 875-885, 2010.

[22] K. L. Tan, D. W. Scott, F. Hong et al., "Tumor-associated macrophages predict inferior outcomes in classic Hodgkin lymphoma: A correlative study from the E2496 Intergroup trial," Blood, vol. 120, no. 16, pp. 3280-3287, 2012.

[23] P. Farinha, H. Masoudi, B. F. Skinnider et al., "Analysis of multiple biomarkers shows that lymphoma-associated macrophage (LAM) content is an independent predictor of survival in follicular lymphoma (FL)," Blood, vol. 106, no. 6, pp. 2169-2174, 2005.

[24] E. Suyani, G. T. Sucak, N. Akyürek et al., "Tumor-associated macrophages as a prognostic parameter in multiple myeloma," Annals of Hematology, vol. 92, no. 5, pp. 669-677, 2013.

[25] S. Panchabhai, K. Kelemen, G. Ahmann, S. Sebastian, J. Mantei, and R. Fonseca, "Tumor-associated macrophages and extracellular matrix metalloproteinase inducer in prognosis of multiple myeloma," Leukemia, vol. 30, no. 4, pp. 951-954, 2016.

[26] X. Chen, J. Chen, W. Zhang et al., "Prognostic value of diametrically polarized tumor-associated macrophages in multiple myeloma," Oncotarget , vol. 8, no. 68, pp. 112685-112696, 2017.

[27] C. E. Green, T. Liu, V. Montel et al., "Chemoattractant signaling between tumor cells and macrophages regulates cancer cell migration, metastasis and neovascularization," PLoS ONE, vol. 4 , no. 8, 2009.

[28] L. F. Porrata, K. Ristow, T. M. Habermann et al., "Absolute monocyte/lymphocyte count prognostic score is independent of immunohistochemically determined cell of origin in predicting survival in diffuse large B-cell lymphoma," Leukemia \& Lymphoma, vol. 53, no. 11, pp. 2159-2165, 2012.

[29] R. A. Wilcox, K. Ristow, T. M. Habermann et al., "The absolute monocyte count is associated with overall survival in patients newly diagnosed with follicular lymphoma," Leukemia \& Lymphoma, vol. 53, no. 4, pp. 575-580, 2012.

[30] J.-J. Huang, Y.-J. Li, Y. Xia et al., "Prognostic significance of peripheral monocyte count in patients with extranodal natural killer/T-cell lymphoma," BMC Cancer, vol. 13, article 222, 2013.

[31] L. F. Porrata, K. Ristow, J. P. Colgan et al., "Peripheral blood lymphocyte/monocyte ratio at diagnosis and survival in classical Hodgkin/s lymphoma," Haematologica, vol. 97, no. 2, pp. 262-269, 2012. 
[32] A. Romano, N. L. Parrinello, C. Vetro et al., "Prognostic meaning of neutrophil to lymphocyte ratio (NLR) and lymphocyte to monocyte ration (LMR) in newly diagnosed Hodgkin lymphoma patients treated upfront with a PET-2 based strategy," Annals of Hematology, pp. 1-10, 2018.

[33] L. Saeed, M. M. Patnaik, K. H. Begna et al., "Prognostic relevance of lymphocytopenia, monocytopenia and lymphocyteto-monocyte ratio in primary myelodysplastic syndromes: A single center experience in 889 patients," Blood Cancer Journal, vol. 7, no. 3, 2017.

[34] A. Romano, N. L. Parrinello, C. Cerchione et al., "The NLR and LMR ratio in newly diagnosed MM patients treated upfront with novel agents," Blood Cancer Journal, vol. 7, no. 12, 2017.

[35] T. Dosani, F. Covut, R. Beck, J. J. Driscoll, M. de Lima, and E. Malek, "Significance of the absolute lymphocyte/monocyte ratio as a prognostic immune biomarker in newly diagnosed multiple myeloma," Blood Cancer Journal, vol. 7, no. 6, p. e579, 2017. 


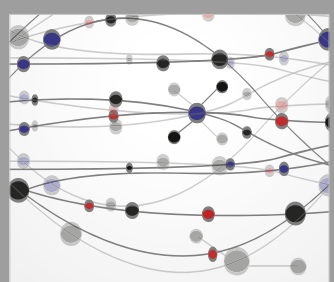

The Scientific World Journal
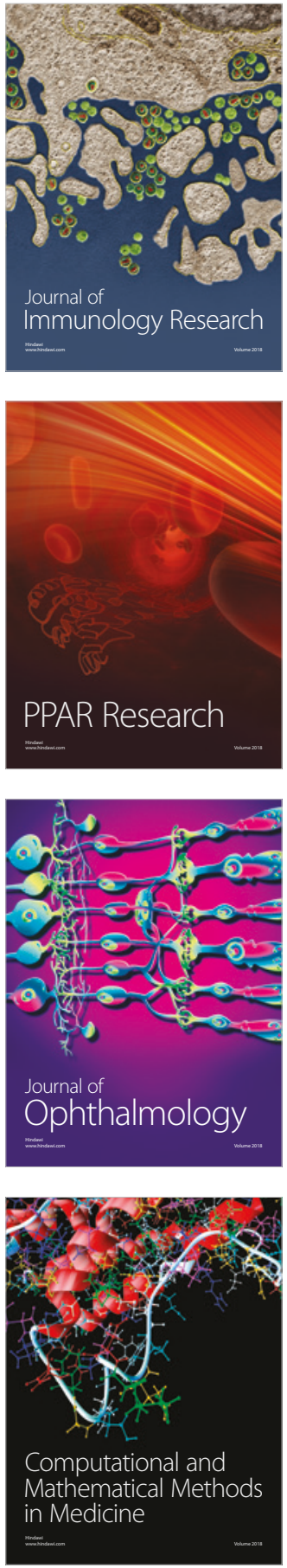

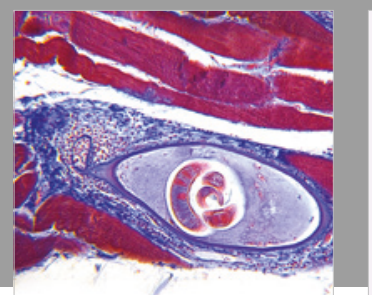

Gastroenterology Research and Practice

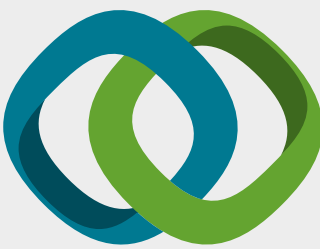

\section{Hindawi}

Submit your manuscripts at

www.hindawi.com
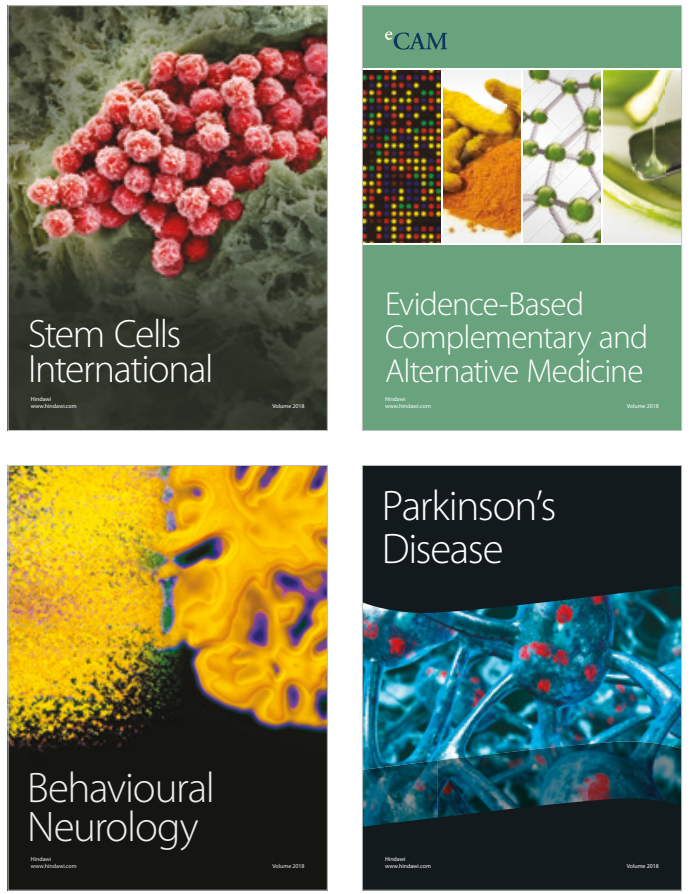

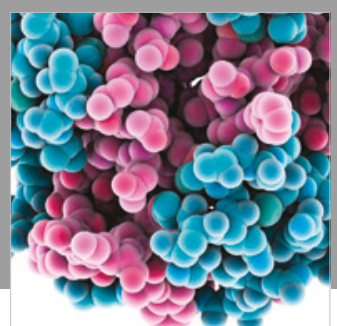

ournal of

Diabetes Research

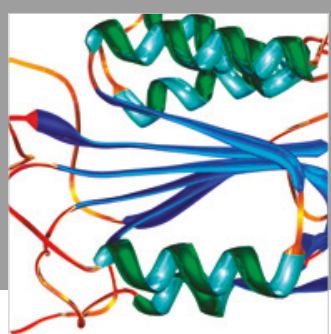

Disease Markers
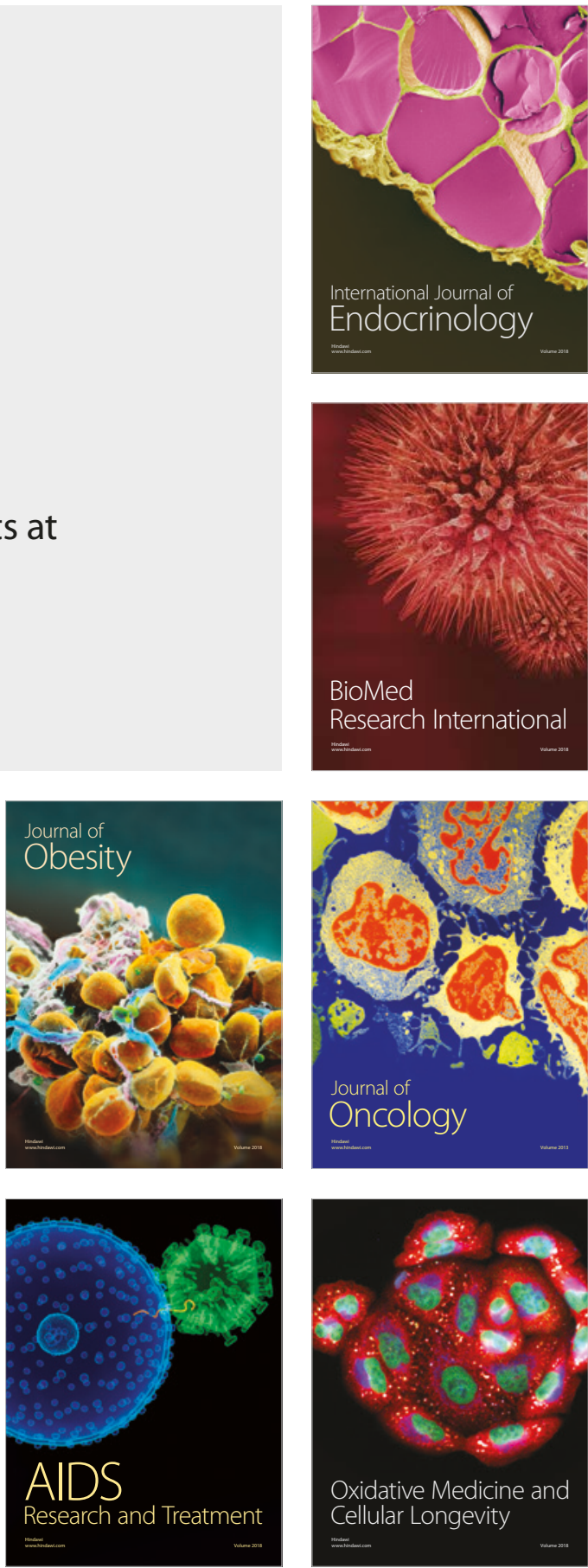